\title{
Synchronous Bilateral Breast Cancer (SBBC): Report of a Case with Review of Literature
}

\author{
Mazaher Ramezani ${ }^{1}$, Akram Amiri ${ }^{2}$, Farhad Kavousi ${ }^{1}$, Masoud Sadeghi, ${ }^{3, *}$ \\ ${ }^{1}$ Molecular Pathology Research Center, Emam Reza Hospital, Kermanshah University of Medical Sciences, Kermanshah, Iran \\ ${ }^{2}$ Students Research Committee, Kermanshah University of Medical Sciences, Kermanshah, Iran \\ ${ }^{3}$ Medical Biology Research Center, Kermanshah University of Medical Sciences, Kermanshah, Iran \\ *Corresponding author: Sadeghi_mbrc@yahoo.com
}

\begin{abstract}
The incidence of synchronous bilateral invasive breast cancer (SBBC) is reported to range from 0.8\%-3.0\%. Lower disease-free survival and high rates of distant metastasis is a recognized feature of bilateral synchronous tumors, which therefore have a worse overall survival compared to unilateral tumors. Early detection of the contralateral tumor is of utmost importance emphasizing the significance of breast self-examination. Here we report a case of SBBC with invasive ductal carcinoma on both sides in a 50-year-old lady. The pathology report on both sides was infiltrating ductal carcinoma and ductal carcinoma in situ, nuclear grade of II/III and histologic grade of II/III. Perineural invasion was present on both sides but vascular invasion only on the right side. In conclusion, SBBC is rare, but is important because it may have worse prognosis, different hormone receptor status and treatment modality. Early detection with breast self-examination is highly emphasized.
\end{abstract}

Keywords: synchronous, bilateral, breast cancer, case report

Cite This Article: Mazaher Ramezani, Akram Amiri, Farhad Kavousi, and Masoud Sadeghi, "Synchronous Bilateral Breast Cancer (SBBC): Report of a Case with Review of Literature.” American Journal of Medical Case Reports, vol. 5, no. 12 (2017): 301-303. doi: 10.12691/ajmcr-5-12-5.

\section{Introduction}

Bilateral breast cancer (BBC) incidence is estimated to be between 0.3 to $12 \%$ [1]. It is categorized as synchronous bilateral breast cancer (SBBC) or metachronous bilateral breast cancer (MBBC). The incidence of SBBC is $1 \%$ and that of MBBC $7.0 \%$ [2]. Different publications suggest the same time to the same year for considering SBBC. Most of the publications proposed the contralateral tumor that was detected within the first 6 months as SBBC, tumors that developed after 6 months as MBBC [3]. The incidence of SBBC is reported to range from $0.8 \%-3.0 \%$; largely reflecting differing definitions used for synchronous diagnoses, with cutoffs ranging from within 3-12 months of initial diagnosis [4]. Synchronicity/ meta chronicity are usually associated with local and lymphatic spread and with blood-borne spread to lungs, bones, and liver [5]. $\mathrm{SBBC}$ is an uncommon presentation, and the management of patients with this disease is not well established. Lower disease-free survival and high rates of distant metastasis is a recognized feature of bilateral synchronous tumors, which therefore have a worse overall survival compared to unilateral tumors [6]. In SBBC patients, molecular analysis showed discordant mutations in all cases, supporting the diagnosis of de novo bilateral primary BCs. In patients with lymph node metastases, the primary BC and metastases shared the same mutations, revealing a metastatic lesion [7]. Contradictory data exists concerning the prognosis of patients with SBBC. Most authors report a worse prognosis for SBBC patients compared to unilateral BC patients. There are a few studies that did not support these findings [8]. The data indicate that multifocal (MF), multicentric (MC), and SBBCs are more aggressive and have an equivalent or moderately poorer survival rate compared with unilateral cases [9]. Here we report a case of SBBC with invasive ductal carcinoma on both sides in a 50-year-old lady.

\section{Case Report}

A 50-year-old lady was admitted in surgery department with chief complaint of bilateral palpable breast masses since 6 months ago on June $7^{\text {th }}, 2016$. The masses were non-tender and without discharge. She was a housewife, married and was living in a village near Harseen, a city in Kermanshah province, Iran. She was the first of the seven sisters. There was no family history except for cystic lesion of the breast in the $4^{\text {th }}$ sister without any treatment or follow-up. There was no past medical or drug history. She was not smoker or opium addict. Physical examination was unremarkable except for bilateral breast masses measuring about 5.5 and $5 \mathrm{~cm}$ in right and left breasts, respectively. The patient underwent surgery on June $9^{\text {th }} 2016$ with frozen section study. On the right side, the gross specimen consisted of a piece of fibro-fatty tissue measuring $5 * 4 * 1.5 \mathrm{~cm}$ containing two firm masses measuring 2.5 and $1.6 \mathrm{~cm}$ with fatty tissue invasion. On the left side, the gross specimen consisted of a piece of fibro-fatty tissue measuring $4 * 3 * 1 \mathrm{~cm}$ with a firm creamy 
yellow mass measuring $2.5 \mathrm{~cm}$. Then bilateral mastectomy and lymph node dissection were done in the same session. The pathology report on both sides was infiltrating ductal carcinoma and ductal carcinoma in situ, nuclear grade of 2/3 and histologic grade of II/III (Figure 1 \& Figure 2). In situ component was $30 \%$ versus $60 \%$ of surface area in right and left sides respectively. The diameter of tumors on the right side was $4.1 \mathrm{~cm}$ and on the left was $2.5 \mathrm{~cm}$. Perineural invasion was present on both sides but vascular invasion only on the right side. On the mastectomy specimens the remnant of the right breast showed infiltrating ductal carcinoma $(0.6 \mathrm{~cm})$ and ductal carcinoma in situ, but in the left side, only remnant of ductal carcinoma in situ (low grade, $0.55 \mathrm{~mm}$ ) was present. The left side showed fibrocystic change and fibroadenoma. Sentinel lymph node of the right and nine isolated lymph nodes of the left axillae were free of tumor. Surgical margins were free of tumor. The patient was in good condition in her admission for the $5^{\text {th }}$ course of chemotherapy on October $6^{\text {th }}, 2016$ and there was only non-productive cough in her history and physical examination.

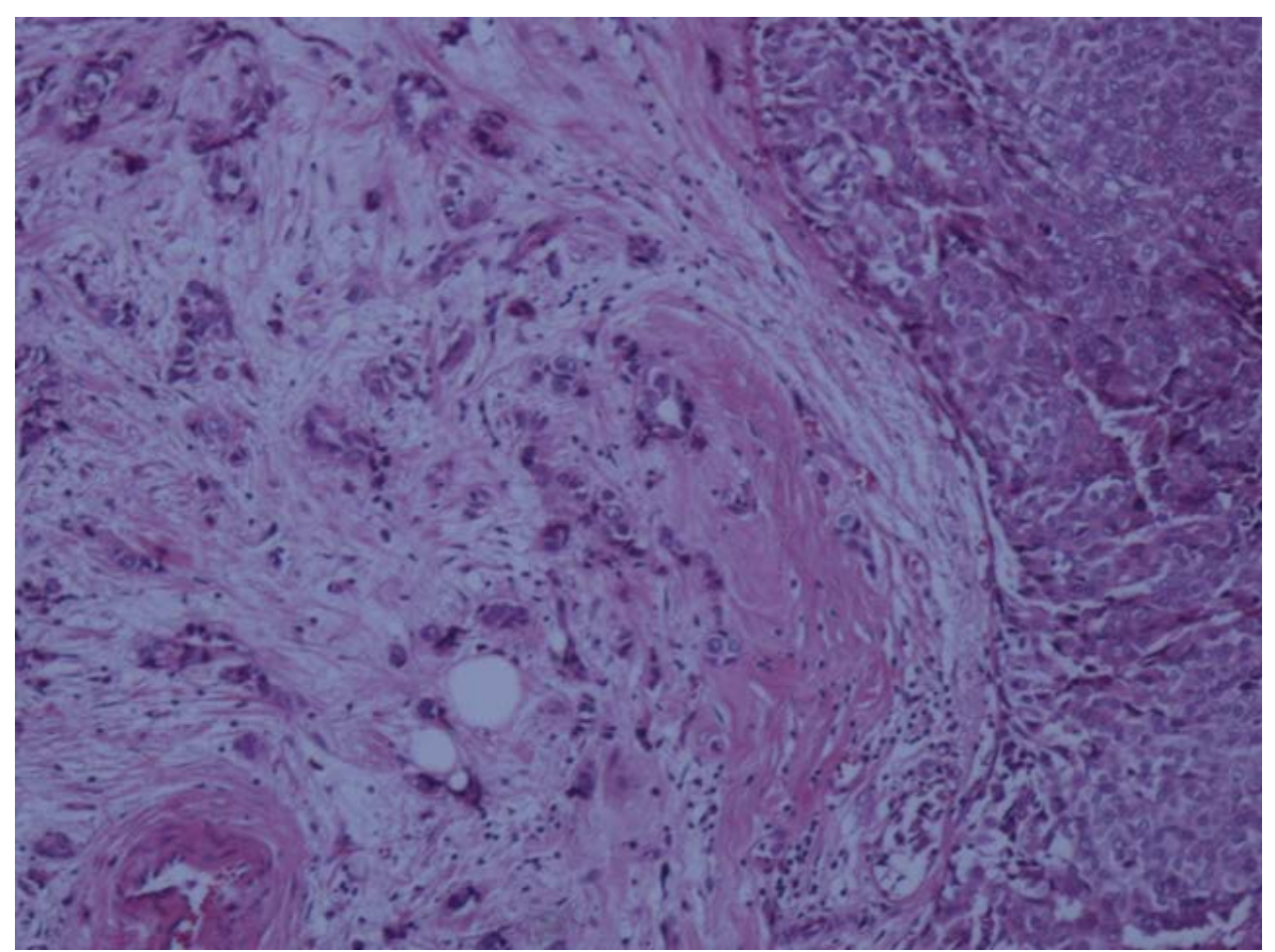

Figure 1. Right breast infiltrating ductal carcinoma and ductal carcinoma in situ, Hematoxylin-Eosin stain (x100)

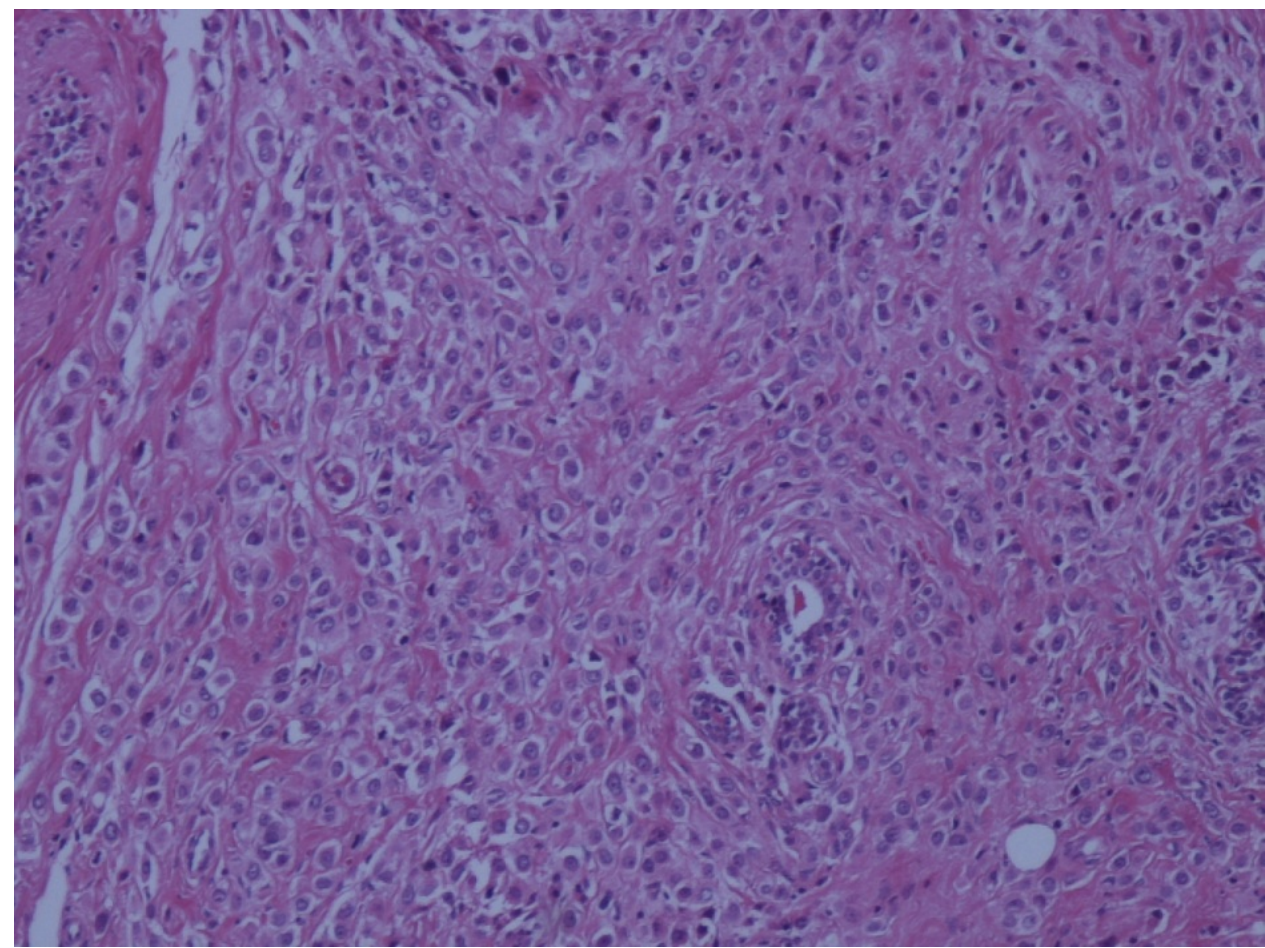

Figure 2. Left breast infiltrating ductal carcinoma, Hematoxylin-Eosin stain (x100) 


\section{Discussion}

Early detection of the contralateral tumor is of utmost importance emphasizing the significance of breast self-examination [5]. Moreover, BBC is mostly lobular carcinomas, but Kappikeri and Kriplani reported a 56-year-old lady with infiltrating ductal carcinoma (IDC) as the primary carcinoma and lobular carcinoma as the secondary [5]. Manea et al. [1] reported SBBC in a young patient complicated in its evolution by ovarian metastases. This form of metastasis is rare in young women and occurs in advanced stages of the disease. Jobsen et al. [2] noted MBBC showed a significant higher local failure compared to UBC and SBBC compared to MBBC and UBC had a significant higher distant metastases rate [3], disease-specific survival and OS were significantly worse for SBBC compared to UBC and MBBC [4], and the OS for MBBC compared to UBC, was significantly better [2].

In the study of Meltem Baykara et al. [3] metachronous and synchronous groups showed similar age, menopausal status, tumor type, HER2/neu expression; but the family history, tumor grade, tumor stage, ER-negativity rate, local and distant metastases rates, surgery, adjuvant chemotherapy application rates were identified as significantly different. Although BBC is not frequent, MCBCis different from MFBC by having more advanced grade, stage, less ER expression, more frequent rates of local relapse and distant metastasis and better response to chemotherapy in case of relapse/metastasis [3]. Another study [4] demonstrated that among women with synchronous bilateral ER+/HER2- BC, recurrence score were concordant in $67 \%$ of cases. Concordance rates may be higher in older women or among those with comparable levels of PR expression. These data suggest that testing of both tumors should be considered in patients who are candidates for adjuvant chemotherapy.

There are no consistent evidence-based guidelines for the management of SBBC and treatment decisions are often made based on the primary tumor with the most high-risk features (i.e., hormone receptor status, HER2 status, size, grade, lymphovascular invasion, and others) [4]. Esclovon et al. [10] reported a 59 years old woman with ER and PR negativity and HER2 /neu positivity on the right breast mass and the opposite results on the incidentally found left breast invasive ductal carcinoma. This different biological behavior mandates different treatment modalities for each side. Schmid et al. [8] compared 46 SBBC with 100 unilateral BCs and found no significant difference in disease -specific survival. Another group demonstrated that the five-year survival rates and recurrence rates were not statistically different among three groups of multifocal, unilateral multi centric and bilateral synchronous tumors [9]. Ursaru and colleagues [11] concluded that patients in the 4th decade diagnosed with unilateral BC are at risk of developing BBC. Piero et al. [12] reported a 44-year-old woman presenting SBBC and characteristic mucocutaneous lesions of Cowden syndrome, confirmed by PTEN gene mutation analysis. The skin lesions showed the characteristic findings of tricholemmoma and concluded that SBBC with early onset suggests a hereditary predisposition. Thus, analysis of PTEN expression abnormality, easily assessed by immunohistochemistry, may be of clinical value to screen those patients with CS. Sostaric Zadro and the colleagues [13] reported a 79-year-old lady with SBBC of invasive ductal carcinoma who responded to a bilateral mastectomy, lymphadenectomy, and radiotherapy.

\section{Conclusion}

SBBC is rare, but is important because it may have worse prognosis, different hormone receptor status and treatment modality. Early detection with breast selfexamination is highly emphasized.

\section{Acknowledgments}

The help of Mrs. Sholeh Akradi in providing the clinical history of the patient is highly acknowledged by the authors.

\section{References}

[1] Manea E, Munteanu A. Evolution of synchronous bilateral breast carcinoma in a young patient. Rev Med Chir Soc Med Nat Iasi. 2016; 120(1): 192-6.

[2] Jobsen JJ, van der Palen J, Ong F, Riemersma S, Struikmans H. Bilateral breast cancer, synchronous and metachronous; differences and outcome. Breast Cancer Res Treat. 2015; 153(2): 277-83.

[3] Baykara M, Ozturk SC, Buyukberber S, Helvaci K, Ozdemir N, Alkis N, et al. Clinicopathological Features in Bilateral Breast Cancer. Asian Pacific J Cancer Prev. 2012; 6(9): 4571-5.

[4] Karsten M, Stempel M, Radosa J, Patil S, King TA. Oncotype DX in Bilateral Synchronous Primary Invasive Breast Cancer. Ann Surg Oncol. 2016; 23(2): 471-6.

[5] Kappikeri VKS, Kriplani AM. Bilateral synchronous carcinoma breast- a rare case presentation. Springerplus. 2015;4:193.

[6] Dalal AK, Gupta A, Singal R, Dalal U, Attri AK, Jain P, et al. Bilateral breast carcinoma- a rare case report. J Med Life. 2011; 4(1): 94-6.

[7] Saad RS, Denning KL, Finkelstein SD, Liu Y, Pereira TC, Lin X, et al. Diagnostic and Prognostic Utility of Molecular Markers in Synchronous Bilateral Breast Carcinoma. Mod Pathol. 2008; 21(10): 1200-7.

[8] Schmid SM, Pfefferkorn C, Myrick ME, Viehl CT, Obermann E, Schötzau A, et al. Prognosis of early-stage synchronous bilateral invasive breast cancer. Eur J Surg Oncol. 2011; 37(7): 623-8.

[9] Kadioğlu H, Özbaş S, Akcan A, Soyder A, Soylu L, Koçak S, et al. Comparison of the histopathology and prognosis of bilateral versus unilateral multifocal multicentric breast cancers. World J Surg Oncol. 2014; 12: 266.

[10] Esclovon JW, Ponder M, Aydin N, Misra S. Challenges of treating incidental synchronous bilateral breast cancer with differing tumour biology. BMJ Case Rep. 2016; 2016.

[11] Ursaru M, Jari I, Gheorghe L, Naum AG, Scripcariu V, Negru D. Bilateral breast cancer: diagnosis and prognosis. Rev Med Chir Soc Med Nat lasi. 2016; 120(2): 316-20.

[12] Peiró G, Adrover E, Guijarro J, Ballester I, Jimenez MJ, Planelles $\mathrm{M}$, et al. Synchronous bilateral breast carcinoma in a patient with cowden syndrome: a case report with morphologic, immunohistochemical and genetic analysis. Breast J. 2010; 16(1): 77-81.

[13] Ŝštarić Zadro A, Fudurić J, Frketić I, Miletić M, Zadro Z, Martinac M, et al. Bilateral synchronous breast cancer. Coll Antropol. 2014; 38(3): 1043-5. 